EPJ Web of Conferences 52, 08001 (2013)

DOI: $10.1051 /$ epjconf/20135208001

(C) Owned by the authors, published by EDP Sciences, 2013

\title{
Direct measurements of cosmic rays in space
}

\author{
Roberta Sparvoli ${ }^{1, a}$ \\ ${ }^{1}$ University of Rome Tor Vergata, Italy
}

\begin{abstract}
Direct measurements of the chemical composition and fluxes of cosmic rays have always played a crucial role in advancing our understanding of both acceleration and propagation of cosmic rays. Direct detection is performed with three basic technologies: balloon-borne and satellite-borne detectors, and instruments placed aboard space stations. In this talk I will present the basic principles of direct detection and review the most important measurements made by past and present missions, with a view to future projects.
\end{abstract}

\section{Slides}

The slides of the talk can be found on the website of the symposium ISVHECRI 2012: https://indico.desy.de/conferenceOtherViews.py?view=standard\&confId=4594

a roberta.sparvoli@roma2.infn.it 